\title{
La statistique médicale pour les traitements orthopédiques*
}

Klazien Matter-Walstra, Marcel Widmer, Franziska Schoeni-Affolter, Cornelia Balmer, Andre Busato

* Vous trouvez la version intégrale de cet article sous www.bullmed.ch $\rightarrow$ Archives $\rightarrow 2007 \rightarrow 45$.

Correspondance:

PD Dr A. Busato

Université de Berne

Faculté de Médecine

MEM Center, IEFO Institute

for Evaluative Research

in Orthopaedic Surgery

Stauffacherstrasse 78

CH-3014 Berne

Tél. 0316315962

Fax 0316315961

andre.busato@MEMcenter.unibe.ch
Depuis janvier 1997, la «Statistique médicale des hôpitaux» rassemble sur une base annuelle les données relatives aux patients sortis des établissements hospitaliers de Suisse. On peut ainsi se faire une idée exacte de la situation de la pratique de soins et du recours aux hôpitaux par la population du pays. Dans le cadre de ces études, outre le découpage traditionnel par canton, on a défini des zones liées à un établissement hospitalier. On a ainsi défini dans l'ensemble du pays 612 régions «MedStat», dont 240 comptent au moins un centre hospitalier. Ces zones liées à un centre hospitalier rassemblent plusieurs régions «MedStat» qui combinent au minimum une région et un hôpital. La définition de ces zones s'appuie sur la méthode de la «small area analysis». L'analyse du recours aux prestations hospitalières en Suisse impose le recueil de l'ensemble des prestations médicales (CHOP) et le codage cohérent des actes de diagnostic (ICD-10) et de soins. L'office fédéral de la Statistique attribue le code «0000» aux données CHOP qui n'ont pas été saisies, ce qui peut correspondre aux situations suivantes selon les directives de codage [1]: 1. la prestation pratiquée ne correspond pas à l'une des catégories CHOP prévue pour le codage; 2 . il s'agit d'une prestation diagnostique ou thérapeutique pour laquelle le code CHOP prévu au chapitre 16 n'a pas été considéré comme adapté au cas particulier; 3. il n'existe pas de code CHOP pour la pres-

Figure 1

Répartition en pourcentages des cas orthopédiques «avec CHOP» et ODDS «sans CHOP» selon les zones de recrutement des établissements, corrigée pour l'âge, l'emplacement et le sexe.

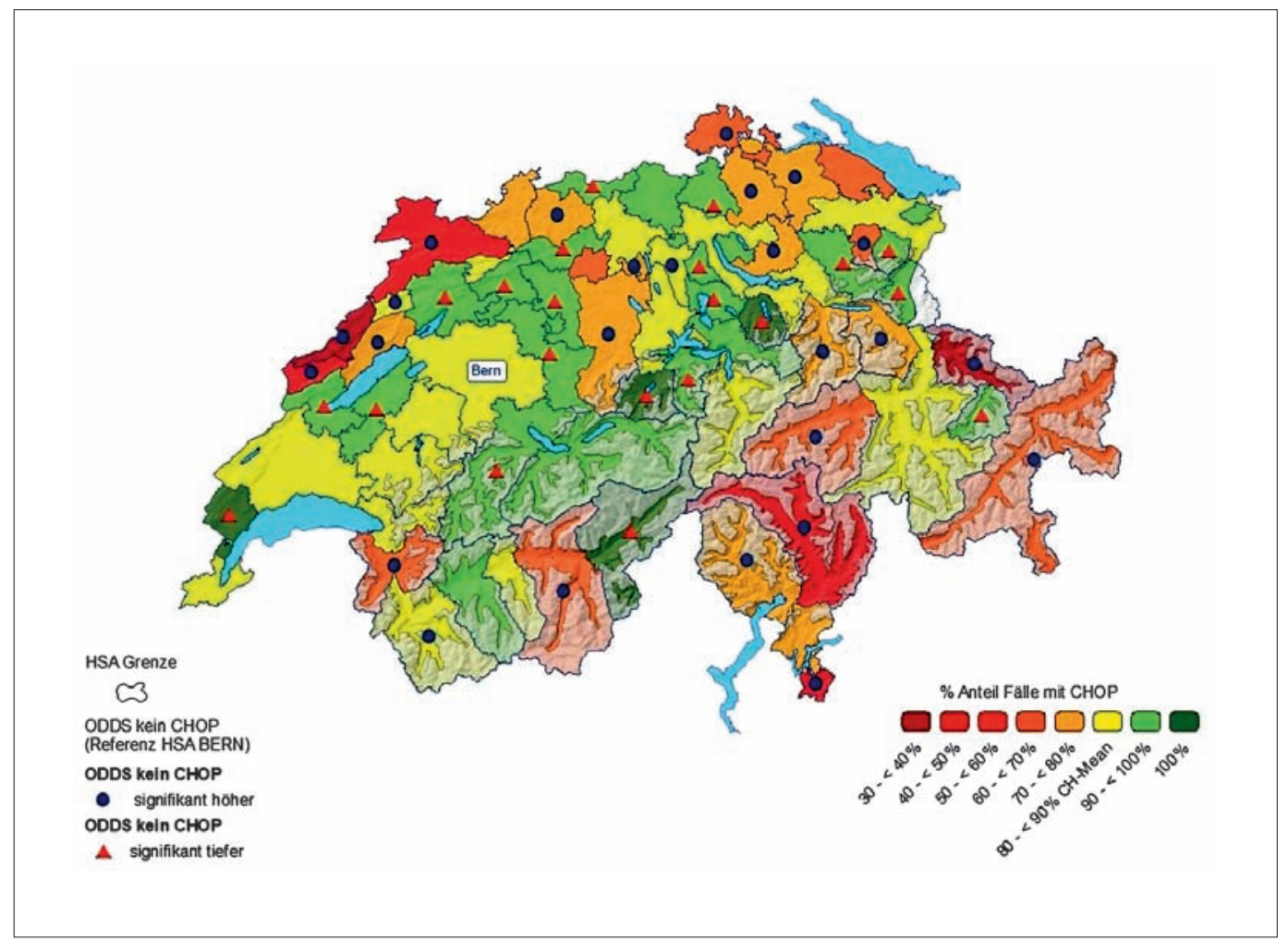


tation appliquée; 4. aucune prestation n'a été pratiquée ou 5. le codage n'a pas été pratiqué («missing»). Les cas où la prestation et/ou le diagnostic n'ont pas été codés sont de nature à entraîner des erreurs d'évaluation de la prestation médicale et à biaiser les analyses statistiques correspondant à une zone considérée («small area analyses»). Dans le contexte du Programme national de recherche «Santé musculo-squelettique - douleurs chroniques» NFP53, l'existence d'un code de l'intervention principale a fait l'objet d'analyse pour les cas qualifiés d'orthopédiques (ceux dont le diagnostic principal relevait des «Maladies de l'appareil musculo-squelettique et des membres» [M00-M99]). L'objectif de cette étude consiste à décrire la méthode de détermination des zones liées à un établissement hospitalier et en outre à déterminer et discuter les effets des facteurs individuels, tels les différences régionales dans la pratique du codage de l'intervention principale, et les conséquences qui en résultent pour l'analyse des «small areas».

Dans le contexte des soins orthopédiques, on peut découper la Suisse en 69 zones liées à un établissement hospitalier. L'analyse a porté sur le codage des soins liés à 216550 cas orthopédiques (diagnostic princeps «M» selon ICD-10, 2002-2003). La conclusion de cette étude est qu'un code d'intervention principale a été attribué au patient lors de sa sortie dans $85 \%$ des cas où le diagnostic principal de «Maladie de l'appareil musculo-squelettique et des membres» avait été porté au cours des années 2002-2003. On notera que cette proportion est en augmentation. A l'échelle du cas individuel, les facteurs susceptibles d'influencer le choix du code principal sont le sexe, l'âge et le lieu d'hospitalisation (hospitalisation dans la zone liée à la résidence du patient, ou en dehors de celle-ci). A l'échelle des établissements hospitaliers, on a identifié un facteur supplémentaire lié à la région d'hospitalisation pour déterminer si un code d'intervention principale a été retenu ou non. Ces différences régionales suscitent la réflexion et pourraient être liées à des facteurs de différenciation tenant à l'interprétation des consignes de codage ou à des incitations particulières. L'analyse des résultats de l'étude du recours aux soins hospitaliers par la technique des zones («small areas analysis») s'en trouve ainsi biaisée. On peut craindre que les différences observées entre les zones considérées ne soient pas liées à des facteurs de disponibilité ou de besoin, mais plutôt à des différences liées à la pratique du codage. L'introduction au niveau national du système forfaitaire en relation avec le cas dénommé SwissDRG (prévue en 2009/2010) devrait contribuer à garantir l'accès à des données plus complètes en relation avec l'intervention principale.

\section{Référence}

1 Office fédéral de la statistique (OFS). Manuel de codage. Neuchâtel: OFS; 2002. 